\title{
Post-mortem CT lung findings at a medicolegal institute in SARS-CoV-2 RT-PCR positive cases with autopsy correlation
}

\author{
Chris O'Donnell ${ }^{1,2} \cdot$ Linda lles $^{1,2} \cdot$ Noel Woodford ${ }^{1,2}$
}

Accepted: 18 May 2021 / Published online: 23 June 2021

○) Springer Science+Business Media, LLC, part of Springer Nature 2021

\begin{abstract}
CoVID-19 is a novel viral infection with now well-established clinical radiological findings. There is limited data on postmortem imaging. We explore the proposition that PMCT could be used as screening test. In an 11-week period, 39 deceased persons were referred for medicolegal investigation with pre-existing or subsequent nasopharyngeal swabs showing positivity on SARS-CoV-2 RT-PCR testing. All 39 had routine whole-body CT scans on admission and 12 underwent medicolegal autopsy. These cases were contrasted with 4 others which were negative on nasopharyngeal swabs despite PMCT findings suggestive of CoVID-19 pneumonia (designated false positive). Nine of the 12 autopsies showed lung histology consistent with those reported in CoVID-19 pneumonia. Typical clinical CoVID-19 lung findings on PMCT were only detected in 5 (42\%). In 3 of the 4 false positive cases, lung findings showed non-COVID-19 histology but in 1, findings were identical. PMCT CoVID-19 findings in the lungs are therefore not specific and may not be detected in all cases due to obscuration by expected agonal CT findings or other pathologies that pre-dated SARS-CoV-2 infection. PMCT findings may otherwise be subtle. Although PMCT may hint at CoVID-19, we believe that nasopharyngeal swabs are still required for definitive diagnosis. Even with positive swabs, clinical CoVID-19 lung findings on PMCT are often not detected. PMCT findings can be subtle, extreme or obscured by agonal changes. Given this range of PMCT changes, the challenge for pathologists is to determine whether death has been caused by, or merely associated with, SARS-CoV-2 infection.
\end{abstract}

Keywords CoVID-19 $\cdot$ Post-mortem computed tomography $\cdot$ Lung $\cdot$ Medicolegal autopsy

\section{Introduction}

Corona viruses are RNA viruses of the family Coronaviridae, widely distributed in animals and humans. In humans they are usually associated with relatively mild symptoms but can be responsible for outbreaks of respiratory illness with high mortality rates including the epidemics of severe adult respiratory syndrome due to SARS-CoV and Middle East respiratory syndrome due to MERS-CoV.

In December 2019, a cluster of pneumonia cases in Wuhan, Hubei, China was shown to be caused by a novel corona virus, since named SARS-CoV-2, causing the disease now known as corona virus disease 2019 (CoVID-19). As

Chris O’Donnell

chris.odonnell@vifm.org

1 Department of Forensic Pathology, Victorian Institute of Forensic Medicine, Southbank, Australia

2 Department of Forensic Medicine, Monash University, Clayton, Australia is well known, the virus has subsequently spread rapidly around the world [1]. On 30 January 2020, the World Health Organization declared a public health emergency of international concern (PHEIC), WHO's highest level of alarm, and subsequently on 11 March 2020 a pandemic was affirmed [2].

Australia had the first case of confirmed CoVID-19 on 25 January 2020 in a Chinese national from Wuhan, China. After an initial community outbreak in Victoria from March to April 2020, followed by small contained clusters, a wave of community-acquired cases developed from July to October 2020 [3]. During this period, the first confirmed case at our Institute was on 5 July 2020 and the last on 22 September 2020 .

Our institute provides forensic pathology services to the Victorian State Coroner. Types of deaths reportable to the Coroner are detailed in the Victorian Coroners Act 2008 and include unexpected death, violent or unnatural death such as homicide; suicide; drug, alcohol and poisons, accident or injury (even if there is a prolonged interval between the 
incident and death); medical procedure or following a medical procedure (where the death is or may be causally related to the medical procedure and a registered medical practitioner would not, immediately before the procedure was undertaken, have reasonably expected the death to occur), Medical Certificate of Cause of Death has not been signed and is not likely to be signed; the identity of the person is unknown; the death occurred in custody or care; or the person was a patient within the meaning of the Mental Health Act 1986 (S. 4).

The role of the coroner (S. 67) is to find the identity, the cause of death, and (if appropriate) the circumstances in which the death occurred. A coroner must direct a medical investigator (pathologist) to perform an autopsy if they believe it is necessary for the investigation of the death (s. 25) [4].

The Victorian Coroners Act 2008 also allows for a socalled Preliminary Examination process that includes a whole-body post-mortem computed tomography (PMCT) scan, performed as a routine procedure on all cases admitted to our Institute. Since 2005, all admitted cases to our Institute have had whole body PMCT imaging [5].

Notwithstanding prior SARS-CoV-2 reverse transcription (RT) - polymerase chain reaction (PCR) positivity, cases were reported to the coroner based on the above parameters. In addition, however, due to the large volume of community transmission, all deceased persons (without pre-existing diagnosis) admitted to the Institute from 11 August through to 31 October 2020, had nasopharyngeal swabs performed by mortuary technicians as part of the admission process and SARS-CoV-2 RT-PCR analysis undertaken. The only exclusions criteria included heavily decomposed bodies and the presence of severe facial trauma (technical limitations), and infants and homicides (due to evidentiary requirements).

\section{Material and methods}

On review of our records, 39 cases were identified that were either known to be SARS-CoV-2 RT-PCR positive on nasopharyngeal swab [6] or were subsequently shown to be positive on admission to the Institute. Demographic details, circumstances of death, past medical history, post-mortem CT appearances and autopsy findings including histology were analyzed.

Table 1 Locations of initial detection of SARS-CoV-2 RT-PCR positivity
Table 2 Results of admission nasopharyngeal swabs for SARS-CoV-2 RT-PCR at our institute

\begin{tabular}{ll}
\hline Result & Number $(\%)$ \\
\hline $\begin{array}{l}\text { negative or } \\
\text { known positive }\end{array}$ & $1336(>99)$ \\
$\begin{array}{l}\text { unexpected posi- } \\
\text { tive }\end{array}$ & $6(<1)$ \\
& $1342(100)$ \\
\hline
\end{tabular}

A further 4 cases of unexpected death from the same time period, that had PMCT features which were suggestive of typical CoVID-19 based on clinical radiological parameters, were reviewed. All 4 had negative SARS-CoV-2 RT-PCR on nasopharyngeal swabs performed after death. These were designated false positive PMCT cases.

PMCT was performed on a dual-source Somatom Definition Flash CT scanner (Siemens, Erlangen, Germany). Standard protocols [7] were employed for each case including whole body examination from head to toe, followed by dedicated smaller field of view (FOV) imaging through the head and neck region. All scans used $120 \mathrm{kV}$ and $128 \times 0.6 \mathrm{~mm}$ collimation with automated dose modulation. Reference mAs in the chest was $280 \mathrm{mAs}$ with reconstruction as $1.5 \mathrm{~mm}$ slices in $1 \mathrm{~mm}$ increments. All chest images were subsequently reconstructed using a smaller FOV to incorporate only the chest wall and a B70f (edge enhancement) reconstruction kernel. These images were used for dedicated radiographic analysis of lung parenchyma and reviewed by a necroradiologist with 15 years experience.

PMCT findings in the lungs were classified into 8 categories based on established PMCT findings in the chest [8] and published clinical CT findings in CoVID-19 pneumonia [9-11]. These 8 categories are:

1. minimal change of CoVID-19 i.e. peripheral ground glass opacity (GGO)

2. typical change of CoVID-19 i.e. GGO, so-called crazy paving, alveolar consolidation

Table 3 PMCT findings of the lungs in the 39 SARS-CoV-2 RT-PCR positive cases

\begin{tabular}{lll}
\hline Category & Findings & Number $(\%)$ \\
\hline 1 & minimal change of CoVID-19 & $2(5)$ \\
2 & typical change of CoVID-19 & $16(41)$ \\
3 & mixed typical change of CoVID-19 and & $6(15)$ \\
& typical agonal changes \\
4 & typical agonal changes & $4(10)$ \\
5 & uniformly dense (airless) lungs & $2(5)$ \\
6 & gross decomposition & $1(3)$ \\
7 & another condition & $5(13)$ \\
8 & another condition and typical agonal & $3(8)$ \\
Total & changes & $39(100)$ \\
\hline
\end{tabular}

\begin{tabular}{ll}
\hline Location & Number $(\%)$ \\
\hline Community & $20(51)$ \\
Inpatient facility & $13(33)$ \\
Our Institute & $6(15)$ \\
& $39(100)$ \\
\hline
\end{tabular}


Table 4 PMCT findings of the lungs in the 12 SARS-CoV-2 RT-PCR positive cases with autopsy

\begin{tabular}{lll}
\hline Category & Findings & Number $(\%)$ \\
\hline 1 & minimal change of CoVID-19 & $1(8)$ \\
2 & typical change of CoVID-19 & $5(42)$ \\
3 & $\begin{array}{l}\text { mixed typical change of CoVID-19 and } \\
\text { typical agonal changes }\end{array}$ & $0(0)$ \\
& typical agonal changes & $0(0)$ \\
5 & uniformly dense (airless) lungs & $1(8)$ \\
6 & gross decomposition & $1(8)$ \\
7 & another condition & $2(17)$ \\
8 & another condition and typical agonal & $2(17)$ \\
& changes & $12(100)$ \\
\hline
\end{tabular}

3. mixed typical change of CoVID-19 and typical agonal changes

4. typical agonal changes only

5. uniformly dense (airless) lungs

6. gross decomposition

7. other condition e.g. cancer, cavitating mass or atelectasis

8. other condition and typical agonal changes (i.e. mixed 7 and 4)

Autopsy was performed in 12 of the SARS-CoV-2 RTPCR positive cases and all 4 of the so-called false positive cases, as directed by a coroner in consultation with a forensic pathologist. This direction that an autopsy be performed was made under the authority of the Victorian Coroners Act 2008 [4], after taking the wishes of the deceased's family into consideration in association with provision of advice from a pathologist as to whether a reasonable cause of death was available based on the findings of the preliminary examination, including the results of a PMCT.

Multicavity autopsy was performed using a standardized technique, with histological specimens of the lungs obtained in all cases. Toxicology testing was performed when indicated by case circumstances.

Histological changes in the lungs were classified into 4 categories: These categories were:

1. typical CoVID-19 pneumonia i.e. diffuse alveolar damage (DAD), intra-alveolar protein, hyaline membranes
Table 5 PMCT findings of the lungs in the 4 SARS-CoV-2 RT-PCR negative, false positive cases

\begin{tabular}{lll}
\hline Category & Findings & Number $(\%)$ \\
\hline 2 & $\begin{array}{l}\text { typical change of CoVID-19 } \\
\text { mixed typical change of CoVID-19 } \\
\text { and typical agonal changes }\end{array}$ & $3(75)$ \\
& & $4(25)$ \\
Total & & $4(100)$ \\
\hline
\end{tabular}

2. organizing $\mathrm{DAD} \pm$ fibrosis

3. bacterial pneumonia or bronchopneumonia

4. another lung pathology

Causes of death were classified into categories as indicated by the supervising forensic pathologist. These categories were:

1. CoVID-19 pneumonia

2. trauma with SARS-CoV-2 + ve

3. SARS-CoV-2 + ve in the setting of illicit drug use

4. suicide with SARS-CoV-2+ve

5. another medical condition with SARS-CoV-2 + ve

\section{Results}

The age range of the SARS-CoV-2 RT-PCR positive cases was 15 - 95 years (mean 75 years) with 24 males and 15 females, and the false positive group was $38-56$ years (mean 45 years) with 1 male and 3 females. The age range of the SARS-CoV-2 RT-PCR positive cases that had autopsy was $30-87$ years (mean 69 years) with 7 males and 5 females.

The location of initial SARS-CoV-2 RT-PCR nasopharyngeal swab positivity was predominately in the community and inpatient hospital facility (Table 1). A total of 1336 screening nasopharyngeal swabs were obtained at our institute, of which $6(<1 \%)$ were unexpectedly positive (Table 2).

PMCT lung findings of CoVID-19 pneumonia, typical of those reported on clinical CT, were detected in 24 cases (62\%) albeit $6(15 \%)$ of these had superimposed agonal lung findings (Table 3). Of the 12 autopsy cases, $6(50 \%)$ had these typical findings (Table 4 ). In the 4 so-called false positive cases, all 4 (100\%) had typical changes of CoVID-19
Table 6 Histological findings in the lungs of the 12 SARSCoV-2 RT-PCR positive autopsy cases

\begin{tabular}{lll}
\hline Category & Findings & Number (\%) \\
\hline 1 & $\begin{array}{l}\text { typical CoVID-19 pneumonitis i.e. diffuse alveolar damage (DAD), } \\
\text { intra-alveolar protein, hyaline membranes }\end{array}$ & $7(58)$ \\
2 & organizing DAD \pm fibrosis & $2(17)$ \\
3 & bacterial pneumonia or bronchopneumonia & $1(8)$ \\
4 & another pathology & $2(17)$ \\
Total & & $12(100)$ \\
\hline
\end{tabular}


Table 7 Histological findings in the lungs of the 4 SARS-CoV-2 RTPCR negative, false positive PMCT cases

\begin{tabular}{lll}
\hline Category & Findings & Number (\%) \\
\hline 1 & $\begin{array}{c}\text { diffuse alveolar damage (DAD), intra-alve- } \\
\text { olar protein, hyaline membranes }\end{array}$ & $0(0)$ \\
2 & $\begin{array}{c}\text { organizing DAD } \pm \text { fibrosis } \\
\text { bacterial pneumonia or bronchopneumonia }\end{array}$ & $1(25)$ \\
3 & $\begin{array}{c}\text { another pathology (alveolar haemorrhage/ } \\
\text { oedema and pulmonary oedema) }\end{array}$ & $2(50)$ \\
4 & & $4(100)$ \\
\hline
\end{tabular}

pneumonia based on clinical CT parameters, 1 with superadded agonal lung changes (Table 5).

In 9 of the $12(75 \%)$ autopsy cases, histology revealed changes consistent with CoVID-19 pneumonia. The remaining 3 had non-specific findings and bronchopneumonia (Table 6). In the 3 of the 4 so-called false positive cases with negative SARS-CoV-2 RT-PCR nasopharyngeal swab, histology revealed findings other than those expected in CoVID-19 pneumonia, but 1 had typical diffuse alveolar damage as might be seen in CoVID-19 pneumonia with associated septic pulmonary artery emboli (Table 7).

In 17 of 39 (44\%) cases, the cause of death was given as exclusively CoVID-19 pneumonia, the others having the cause of death listed as SARS-CoV-2 RT-PCR positivity associated with trauma, illicit drug use, suicide, and other medical conditions. (Table 8). Of the 27 non-autopsy cases this exclusive cause of death was given in $11(41 \%)$ (Table 9) and in the 12 autopsy cases, 6 (50\%) as CoVID19 pneumonia exclusively (Table 10). Cause of death in the so-called false positive cases was drug toxicity and non-SARS-CoV-2 infection in $2(50 \%)$ and another medical condition in $2(50 \%)$ (Table 11).

\section{Discussion}

The Coroners Act prescribes the cases that must be presented for examination at our Institute even if death has occurred in a hospital setting or is expected. In our series,

Table 8 Causes of death in all 39 SARS-CoV-2 RT-PCR positive cases

\begin{tabular}{lll}
\hline Category & Findings & Number $(\%)$ \\
\hline 1 & CoVID-19 pneumonia & $17(44)$ \\
2 & trauma with SARS-CoV-2 + ve & $15(38)$ \\
3 & SARS-CoV-2 + ve in the setting of illicit & $1(3)$ \\
& drug use & $2(5)$ \\
5 & suicide with SARS-CoV-2 + ve & $4(10)$ \\
5 & another medical condition with SARS- & $39(100)$ \\
Total & CoV-2 + ve & \\
\hline
\end{tabular}

Table 9 Causes of death in 27 SARS-CoV-2 RT-PCR positive cases with no autopsy

\begin{tabular}{lll}
\hline Category & Findings & Number $(\%)$ \\
\hline 1 & CoVID-19 pneumonia & $11(41)$ \\
2 & trauma with SARS-CoV-2 + ve & $12(44)$ \\
3 & $\begin{array}{l}\text { SARS-CoV-2 + ve in the setting of illicit } \\
\text { drug use }\end{array}$ & $0(0)$ \\
4 & suicide with SARS-CoV-2 + ve & $1(4)$ \\
5 & another medical condition with SARS- & $3(11)$ \\
& CoV-2 + ve & $27(100)$ \\
\hline
\end{tabular}

33 of 39 deceased persons referred to the VIFM were known to be infected with SARS-CoV-2 by way of previous positive nasopharyngeal swab results. Of these deaths, $20(60 \%)$ were in the community and $13(40 \%)$ in hospital. Given the high incidence of SARS-CoV-2 RT-PCR positivity in the community during this study period, the Institute adopted a policy of routine nasopharyngeal swabbing of all unknown CoVID-19 admissions, of which 6 previously undiagnosed cases returned positive results for SARS-CoV-2. Those cases included blunt head injury (2 cases), chronic illicit drug use, suicide, unexpected death in a person with a CoVID-19 positive partner, and sudden unexpected death with a preceding acute respiratory distress. Three of these cases had an autopsy performed. Two had findings consistent with CoVID-19 pneumonitis but 1 acute head injury case had no specific histological features raising the probability that SARS-CoV-2 infection was coincidental rather than causative of the death.

PMCT has become an important component of medicolegal death investigation as well as the investigation of hospital-related death where autopsy is not available or obtainable due to familial objection [5]. It is routinely performed in all cases admitted to our Institute. Given occupational safety concerns about the potential of infection of mortuary staff and pathologists [12], it has been suggested that PMCT with an appropriate clinical history, could be a useful alternative to autopsy in the absence of

Table 10 Causes of death in 12 SARS-CoV-2 RT-PCR positive cases with autopsy

\begin{tabular}{lll}
\hline Category & Findings & Number $(\%)$ \\
\hline 1 & CoVID-19 pneumonia & $6(50)$ \\
2 & trauma with SARS-CoV-2 + ve & $3(25)$ \\
3 & SARS-CoV-2 + ve in the setting of illicit & $1(8)$ \\
& drug use & $1(8)$ \\
4 & suicide with SARS-CoV-2 + ve & $1(8)$ \\
5 & another medical condition with SARS- & $12(100)$ \\
& CoV-2+ ve & \\
\hline
\end{tabular}


Table 11 Causes of death in the 4 SARS-CoV-2 RT-PCR negative, false positive PMCT cases

\begin{tabular}{ll}
\hline Findings & Number $(\%)$ \\
\hline $\begin{array}{l}\text { Drug toxicity and infective condition (not SARS- } \\
\text { CoV-2) }\end{array}$ & $2(50)$ \\
$\begin{array}{l}\text { Another medical condition (diabetes and coronary } \\
\text { artery disease) }\end{array}$ & $2(50)$ \\
& $4(100)$ \\
\hline
\end{tabular}

nasopharyngeal swab for SARS-CoV-2 RT-PCR testing. This concept of chest CT being used as screening test in CoVID-19 has been explored in the clinical environment but in general has been rejected by multiple eminent societies on the basis of non-specificity of CT, the potential burden on radiology departments and challenges for infection control [13-16] although there are advocates. Their major concern is a small but definite number of cases with initial negative SARS-CoV-2 RT-PCR testing on nasopharyngeal swabs in high-risk individuals, having typical CoVID-19 pneumonitis findings on clinical CT and positive RT PCR on repeat nasopharyngeal testing [17]. To date there has been limited literature published on PMCT findings of CoVID-19 pneumonitis [18-21], and only 1 study of 5 cases looking specifically at the question of PMCT screening for CoVID-19 disease in unexpected community deaths [22].

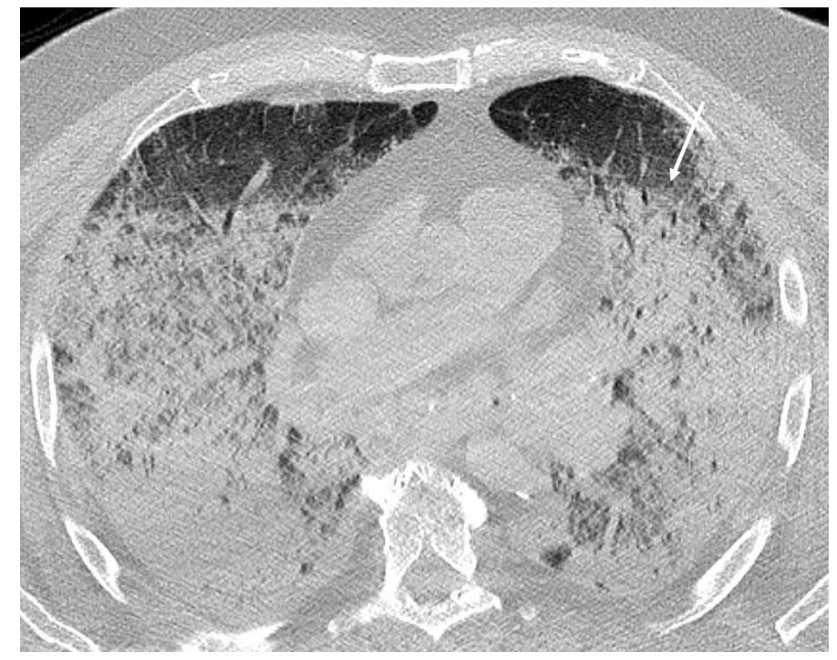

Fig. 2 Axial PMCT in a SARS-CoV-2 RT-PCR positive deceased person, showing more substantial typical findings in CoVID-19 including "crazy paving" and consolidation with air bronchograms (arrow)

Clinical CT in CoVID-19 pneumonitis has well-established findings of predominately peripheral ground glass opacity (GGO), progressing to thickening of interlobular septa ("crazy paving") and eventually dense consolidation with or without air bronchograms, often in a dependent location [9-11]. These findings are stereotypical in CoVID-19 pneumonitis
Fig. 1 a Axial PMCT in a SARS-CoV-2 RT-PCR positive deceased person, showing typical findings in CoVID-19 including GGO and "crazy paving" (arrow). b $\mathrm{H}$ and $\mathrm{E}$ stain $\times 100$ of the lung showing diffuse alveolar damage (DAD) in the exudative phase including hyaline membranes lining alveoli. c $\mathrm{H}$ and $\mathrm{E}$ stain $\times 200$ of the lung showing diffuse alveolar damage (DAD) in the exudative phase including hyaline membranes lining alveoli. $\mathbf{d ~} \mathrm{H}$ and $\mathrm{E}$ stain $\times 200$ of the lung showing Type 2 pneumocyte hyperplasia and fibrin within alveoli
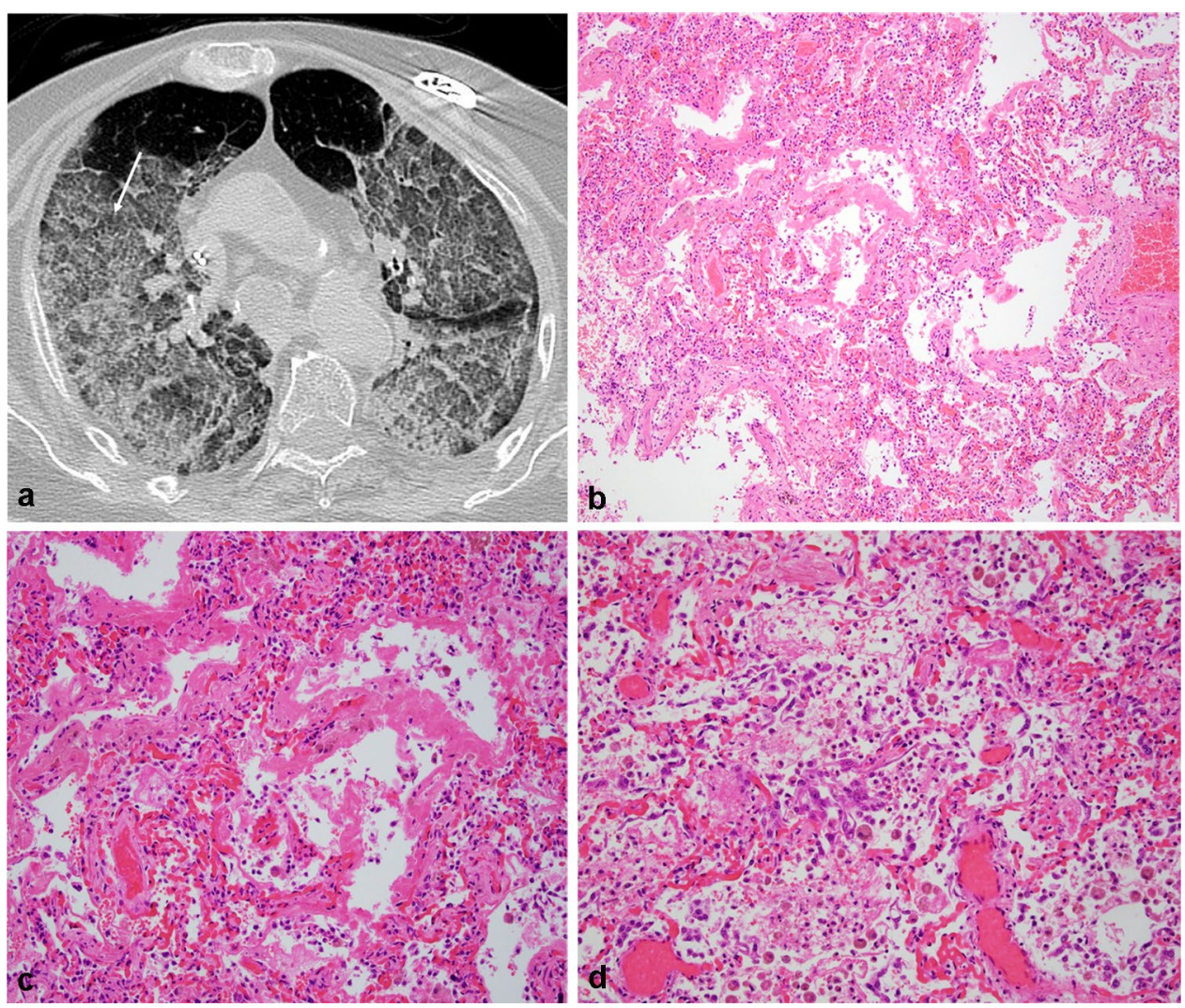
Fig. 3 a Axial PMCT in a SARS-CoV-2 RT-PCR positive deceased person, showing only minimal GGO in the right lung posteriorly (arrow).b volume rendered PMCT showing normally aerated lung apart from minor inflammatory changes in right lower lung (arrow)
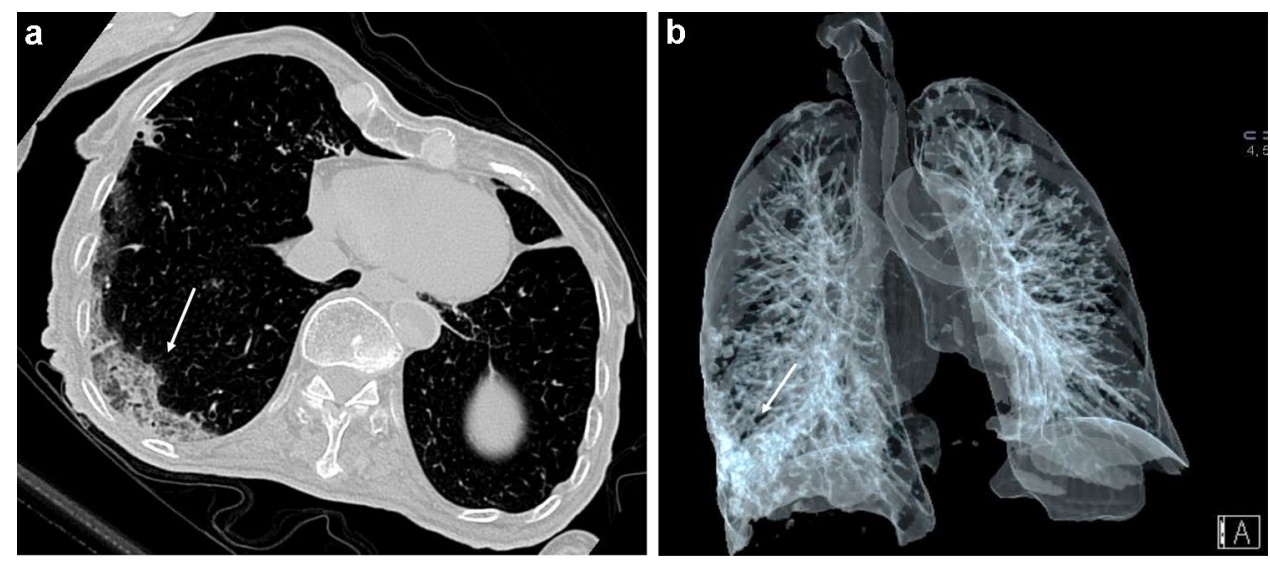

especially the peripheral distribution of early GGO changes albeit overlapping findings seen in other viral pneumonias. Nonetheless clinical radiologists when tested, can distinguish CoVID-19 pneumonitis from other viral pneumonia on chest CT with moderate to high accuracy [23]. Similar patterns may be discernible on PMCT of the chest but need not necessarily be identical to those seen in the living, and may be obscured by known artefacts associated with imaging of the deceased as well as changes that can occur in the lungs as a part of the agonal process.

Overall in 24/39 (62\%) cases, PMCT showed changes that could be described as typical of the clinical findings of CoVID-19 (some with associated agonal changes) but $15 / 39$ (38\%) did not. Of the 24 PMCT positive cases, only 11 had cause of death prescribed by the pathologist as due to CoVID-19 alone, 8 had trauma with SARS-CoV-2 positivity and 1 had SARS-CoV-2 positivity in the setting of illicit drug use (methamphetamine). Of the 4 remaining cases, 2 were suicides with SARS-CoV-2 positivity and 2 had a cause of death stated as natural or advanced dementia with SARS-CoV-2 positivity. These later 13 cases suggest that the presence of SARS-CoV-2 RT-PCR positivity alone does not necessarily indicate the cause of death as being exclusively CoVID-19, and that even the presence of typical clinical Co-VID-19 PMCT findings does not mean that this is the predominate cause of death i.e. it might be an additional factor contributing to the mechanism of death in trauma, chronic drug use or pre-existing medical conditions or an incidental finding in cases of suicide (notwithstanding the psychological effects of receiving a positive test result, the possible direct effects of SARS-CoV-2 on mood or the psychological effects of lockdown). It should be noted that in 1 of the 2 suicides, diagnosis of SARS-CoV-2 RT-PCR positively was only made at our Institute after death.

Of the $15 / 39$ (38\%) cases with no specific clinical CT findings of CoVID-19 on PMCT, 6 had the assigned cause of death as being exclusively CoVID-19. The reasons why PMCT might be non-diagnostic are complex and could relate to deficiencies in $\mathrm{CT}$ interpretation after death (i.e. artefact, agonal changes or end stage lung disease producing nonspecific findings) or the disease itself whereby there are pathways of death other than pneumonitis such as thromboembolism and multiorgan failure [24]. The remaining cases had other associated conditions including 6 with trauma and SARS-CoV-2 positivity, and 3 had cause of death given as unascertained, dehydration and dementia, and natural causes, indicating that PMCT is not always diagnostic in this condition.

Based on our comparison of PMCT and autopsy in 12 cases, only 5 (42\%) cases had typical clinical CoVID19 findings alone on PMCT associated with typical SARS-CoV-2 associated diffuse alveolar damage and/or
Fig. 4 a Axial PMCT scan in a SARS-CoV-2 RT-PCR positive deceased person, showing left lower lobe atelectasis (thick arrow) and oedema in the right lung in a hypostatic (agonal) pattern (thin arrow). b Axial PMCT through the upper abdomen showing a large left peri splenic haematoma (arrow), confirmed at autopsy
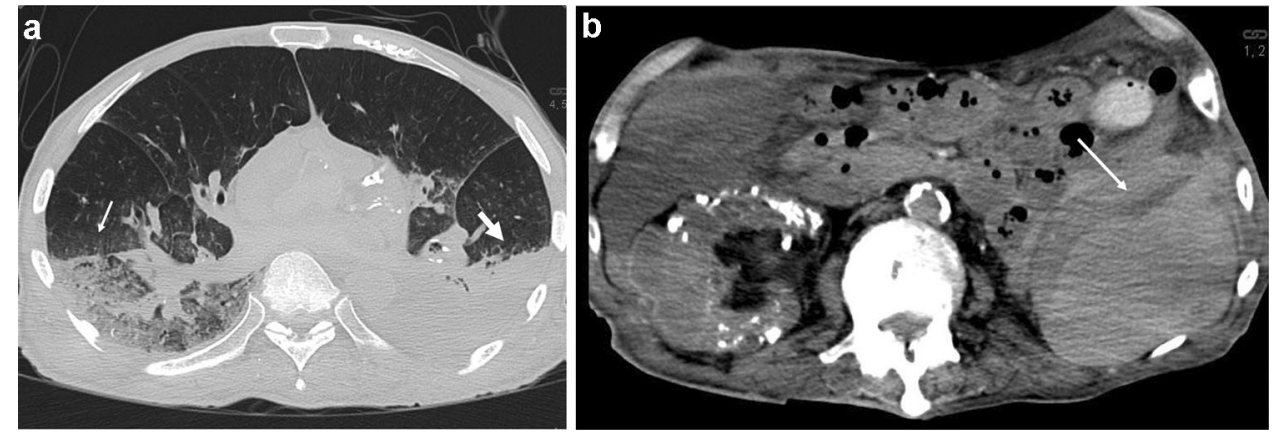


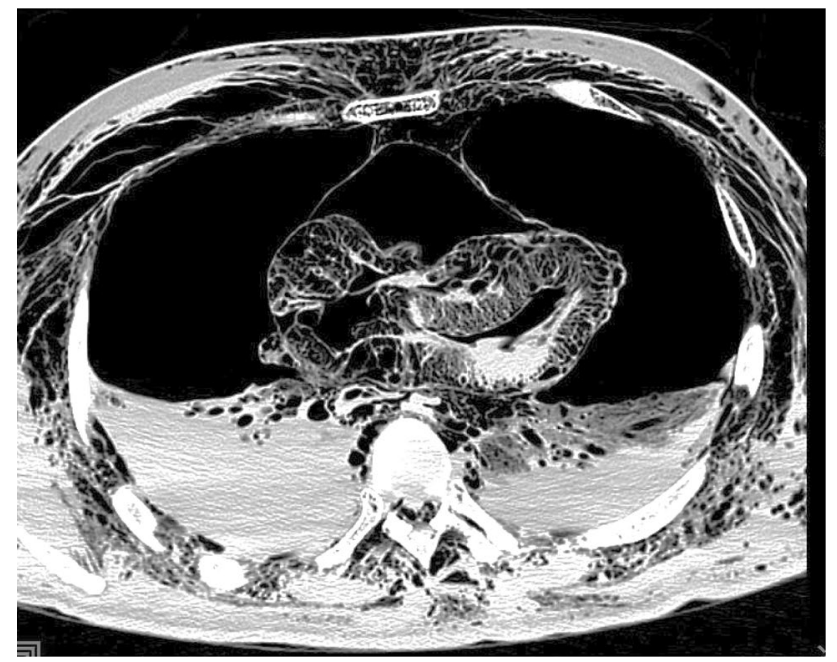

Fig. 5 Axial PMCT in a SARS-CoV-2 RT-PCR positive deceased person, showing gross decompositional gas, pneumothoaces and bilateral pleural fluid. No typical features of CoVID-19 pneumonia

organization and fibrosis at histology (Figs. 1 and 2). The remaining 7 cases are illuminating. PMCT underestimated the effects of SARS-CoV-2 infection due to co-morbidities in one case (Fig. 3) where findings were minimal yet histology showed foci of organizing pneumonia. Interestingly, histology also revealed active chronic bronchiectasis and cause of death was given as complications of advanced dementia with focal bronchiectasis and focal organizing pneumonia due to SARS-CoV-2 infection. This is important information as it reinforces our observation that the presence of SARSCoV-2 RT-PCR positivity in a deceased person is not necessarily the sole causative factor in death. It can be an additional factor, the contribution of which will vary depending on the type and severity of other conditions such as trauma (Fig. 4), pre-existing medical morbidity and illicit drug use.

PMCT interpretation of the lungs is hampered by agonal or artefactual factors obscuring the underlying pathology. This includes agonal aspiration of gastric contents, reduced ventilation, and effects of resuscitation. Artefacts due to post-mortem changes in the chest include hypostasis of lung fluid, pooling and clotting of blood, decomposition and autolysis. In a case with severe decomposition (Fig. 5), the lung findings were obscured by putrefactive gas on CT, yet histology was able to reveal diffuse alveolar damage. In 2 cases, PMCT showed findings that suggested pathology other than CoVID-19. This was confirmed in one as bronchopneumonia but in another PMCT showed typical agonal changes of hypostatic effects and atelectasis, yet histology showed diffuse alveolar damage due to SARS-CoV-2 infection (Fig. 6). In 2 of our cases where histology was considered non-specific, PMCT showed diffuse alveolar opacity and pleural fluid (presumed to be agonal in a fatal head

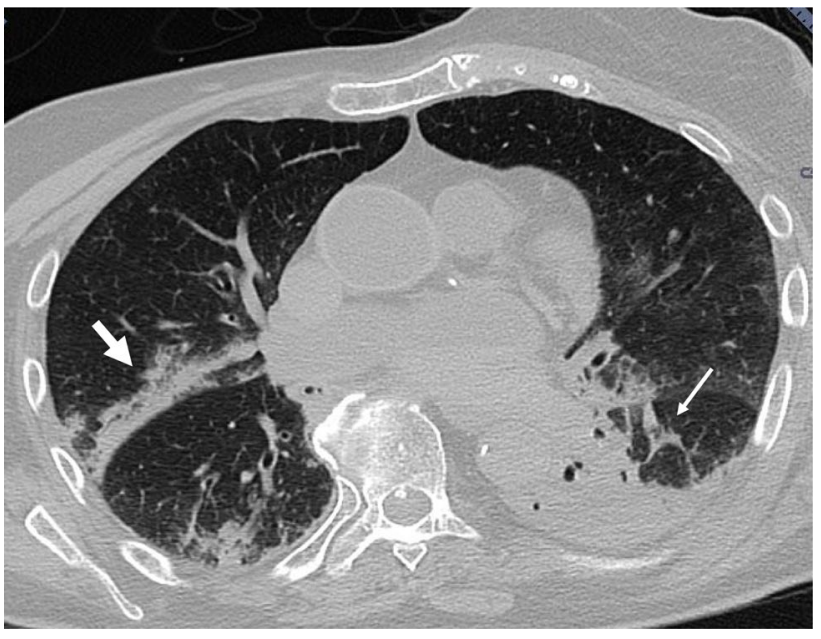

Fig. 6 Axial PMCT in a SARS-CoV-2 RT-PCR positive deceased person, showing left lower lobe atelectasis (thin arrow) and mild alveolar opacity adjacent to the oblique fissure (thick arrow) rather than peripheral GGO

injury) and basal lung atelectasis (presumed to be agonal in a death due to dehydration and dementia).

In addition there may be underlying lung disease or superadded complications of CoVID-19 pneumonitis in life that can further affect PMCT radiological findings. Ultimately the clinically described CT pattern of dense airless lungs due to ground glass opacity and consolidation described in advanced CoVID-19 pneumonitis [9-11], is a common nonspecific pattern seen in many other PMCT cases, where death is due to a respiratory illness leading to a final common pathway of adult respiratory death syndrome (ARDS). In one case with uniformly dense lungs on PMCT, histological changes

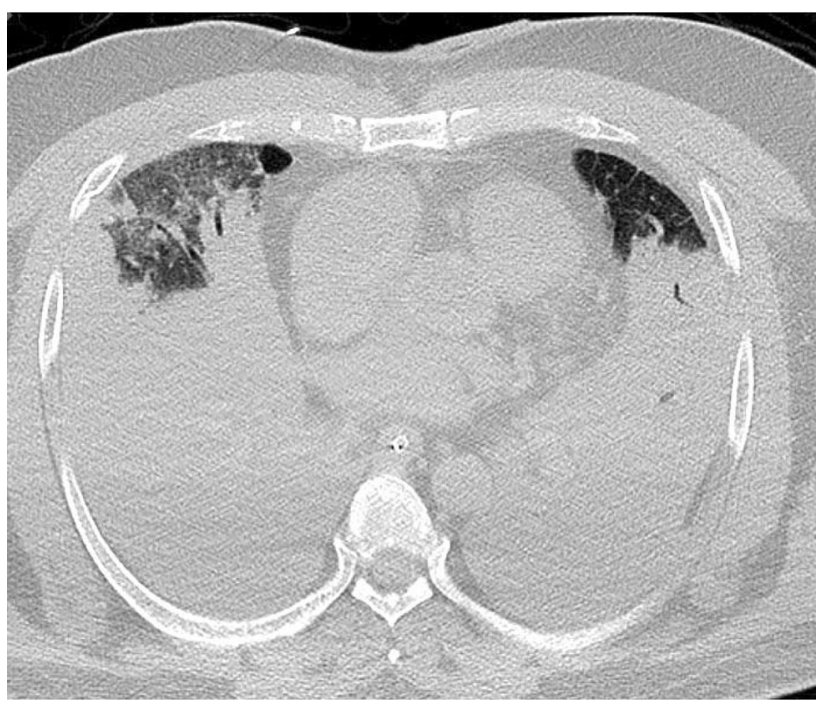

Fig.7 Axial PMCT scan in a SARS-CoV-2 RT-PCR positive deceased person, showing bilateral dense, almost airless lungs 
Fig. 8 a Axial PMCT in a SARS-CoV-2 RT-PCR negative deceased person, showing peripheral GGO, "crazy paving" and air bronchogram (arrow). b $\mathrm{H}$ and $\mathrm{E}$ stain $\times 100$ of the lung showing neutrophils filling alveoli and bronchopneumonia. c $\mathrm{H}$ and $\mathrm{E}$ stain $\times 200$ of the lung showing neutrophils and due to aspiration foreign debris filling airspaces

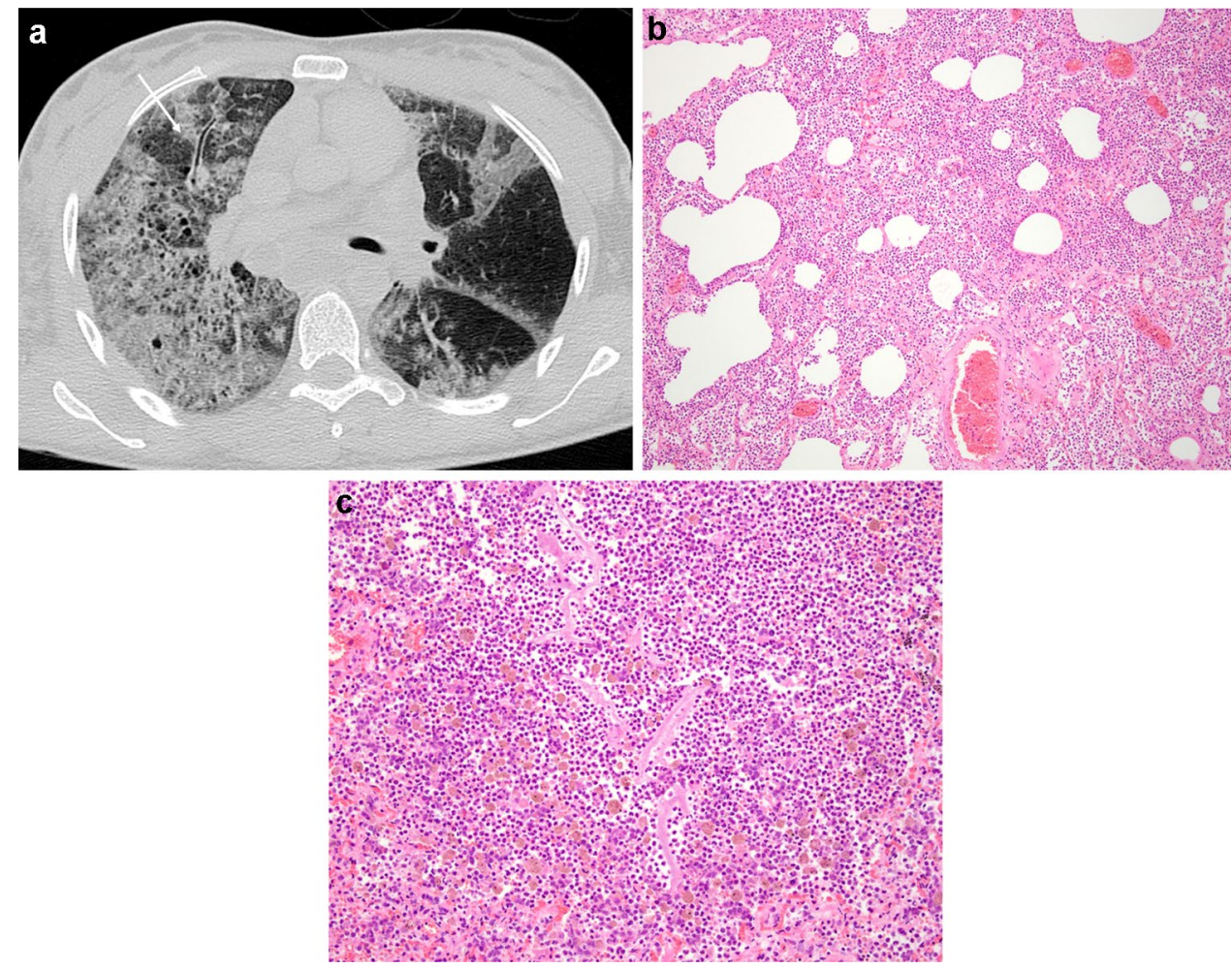

were of SARS-CoV-2 associated diffuse alveolar damage alone without organizing pneumonia or fibrosis (Fig. 7).

Our 4 so-called false positive imaging cases (with negative SARS-CoV-2 RT-PCR nasopharyngeal swab), highlight the relatively non-specific nature of CT lung changes in
CoVID-19 that must be correlated with clinical parameters in the living for a definitive diagnosis. This is not always available in the deceased. In all 4 cases, PMCT findings in the lungs were typical of clinical CoVID-19 pneumonitis, 1 having additional changes seen commonly as an agonal
Fig. 9 a Axial PMCT in a SARS-CoV-2 RT-PCR negative deceased person, showing patchy peripheral GGO and "crazy paving" (arrow). b $\mathrm{H}$ and E stain $\times 100$ of the lung showing hyaline membranes due to the exudative phase diffuse alveolar damage and septic pulmonary emboli. c Verhoeff-Van Gieson stain $\times 40$ of the lung showing neutrophilic vasculitis secondary to septic emboli
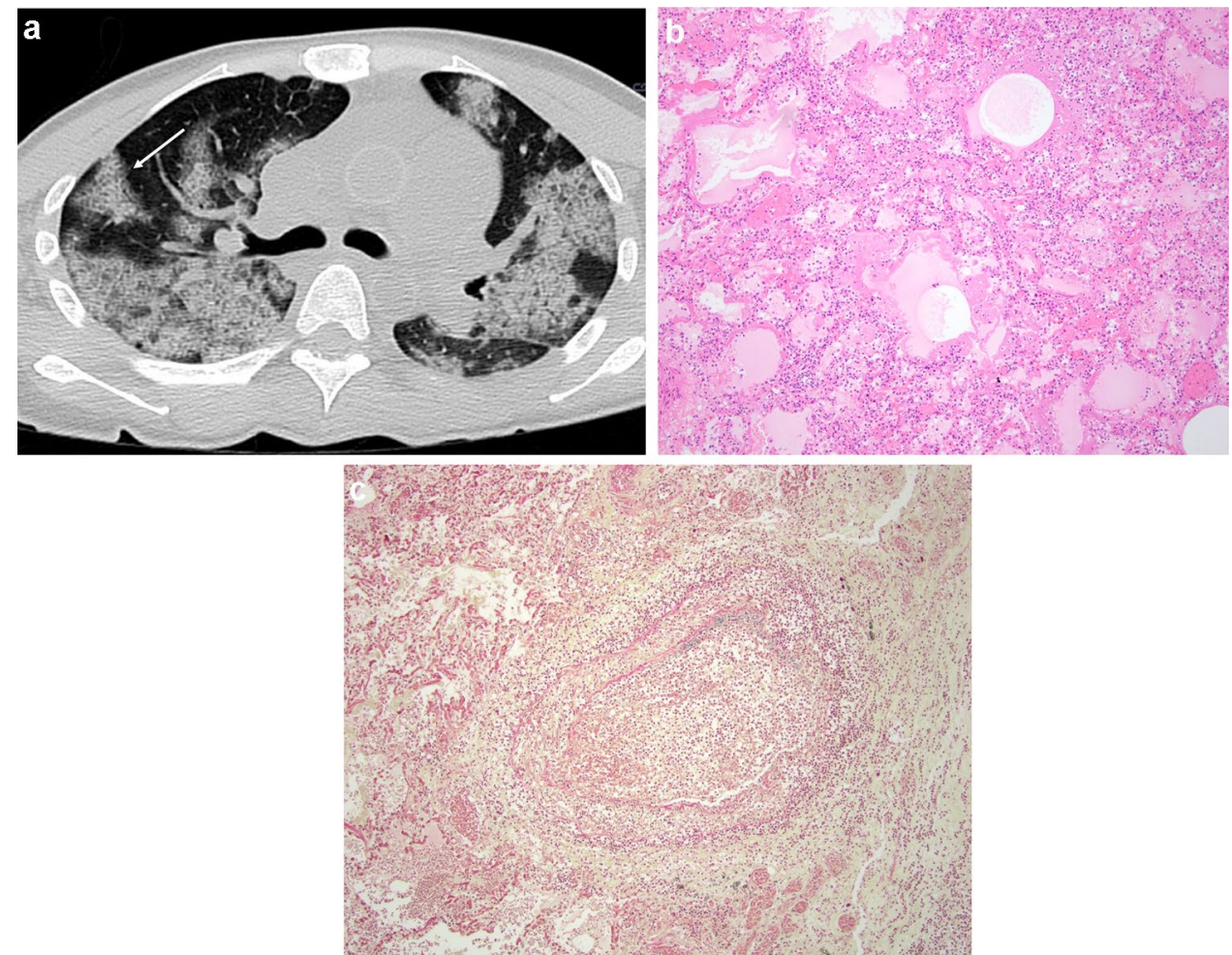
feature on PMCT. Three of these 4 had histological findings that differed dramatically from the diffuse alveolar damage and organizing pneumonia seen in CoVID-19 disease (Fig. 8). The lungs have a limited histological response to pathogens and other pathological processes/insults, explaining why the remaining case with typical PMCT findings also had typical histological changes indistinguishable from those seen in CoVID-19, yet death was due to septic emboli and pulmonary vasculitis in a chronic illicit drug user with infective endocarditis (Fig. 9) rather than CoVID-19.

\section{Conclusion}

The determination that death has been caused by CoVID-19 needs to be accurate from a public health planning perspective and can make an important contribution to contact tracing efforts and quarantining of contacts. Given the demonstrated non-specific findings of CoVID-19 pneumonitis on PMCT in our cohort of both community and hospital related deaths, the absence of major PMCT findings in some cases of SARS-CoV-2 RT-PCR positivity and the obscuring of lung findings by agonal changes in others, we believe that PMCT alone cannot be recommended as a tool for determining cause or manner of death due to CoVID-19 (notwithstanding the local jurisdictional requirements in medicolegal death investigation).

Nonetheless we believe that PMCT can be helpful as a screening tool for CoVID-19 pneumonitis, if the PMCT findings are suggestive and clinical circumstances appropriate. In our Institute, such cases are now escalated to rapid nasopharyngeal swab for SARS-CoV-2 RT-PCR prior to a decision for autopsy (in consultation with the deceased's family). As with all cases at our Institute, autopsy will be undertaken if there are additional specific issues that can only be addressed by internal examination (with staff donning appropriate personal protective equipment) [25]. Otherwise in the absence of any other concerning circumstantial, toxicological, imaging or external examination findings, in our experience PMCT (in association with a positive nasopharyngeal swab) may be considered sufficient in some cases with typical radiological findings, for pathologists to assign cause of death as CoVID-19 pneumonitis, either exclusively or with additional cause(s), satisfying local jurisdictional requirements.

\section{Key points}

1. PMCT findings in CoVID-19 pneumonia may imitate clinical CT findings.
2. Agonal lung changes may obscure PMCT findings in CoVID-19 pneumonia.

3. PMCT findings are not specific for CoVID-19 pneumonia.

4. PMCT findings in SARS-CoV-2 RT-PCR positive cases do not always mimic lung histological changes.

5. Some cases with SARS-CoV-2 RT-PCR positivity on nasopharyngeal swab have no or very minor CoVID-19 changes on PMCT and lung histology, raising the spectre of dying with rather than of the infection.

Acknowledgements The authors would like to thank all forensic pathologists and staff atVIFM who performed the autopsies, mortuary technicians who undertook the PMCTscans and Catherine Vincent, forensic radiographer who post processed the dedicatedCT lung reconstructions. We also wish to acknowledge support from the VictorianState Coroner.

Authors' contributions Chris O'Donnell, concept and principal author, Linda Iles, histology and additional author, Noel Woodford, ethics overview and additional author.

\section{Declarations}

Ethics approval VIFM ethics approval $1176-1158 / 1$ EC 8-2020 dated $27 / 11 / 20$, for project "PMCT findings in SARS-CoV-2 positive persons".

Consent for publication Included in VIFM ethics approval 1176-1158 /1 EC 8-2020.

\section{References}

1. Huang C, Wang Y, Li X, Ren L, Zhao J, Hu Y, et al. Clinical features of patients infected with 2019 novel coronavirus in Wuhan. China Lancet. 2020;395:497-506.

2. Timeline: WHO's COVID-19 response. https://www.who.int/ emergencies/diseases/novel-coronavirus-2019/interactive-timeline. Accessed 1 Apr 2021.

3. Victorian coronavirus (COVID-19) data. https://www.dhhs. vic.gov.au/victorian-coronavirus-covid-19-data. Accessed 1 Apr 2021.

4. Victorian Coroners Act 2008. Act in force, Act Number 77/2008 Version 039. https://www.legislation.vic.gov.au/in-force/acts/ coroners-act-2008/039. Accessed 1 Apr 2021.

5. O'Donnell C, Woodford N. Post-mortem radiology-a new subspeciality? Clin Radiol. 2008;63:1189-94.

6. Zhen W, Manji R, Smith E, Berry GJ. Comparison of four molecular in vitro diagnostic assays for the detection of SARS-CoV-2 in nasopharyngeal specimens. J Clin Microbiol. 2020;58:e00743-e820.

7. Flach PM, Gascho D, Schweitzer W, Ruder TD, Berger N, Ross SG, et al. Imaging in forensic radiology: an illustrated guide for postmortem computed tomography technique and protocols. Forensic Sci Med Pathol. 2014;10:583-606.

8. Filograna L, Thali MJ. Post-mortem CT imaging of the lungs: pathological versus non-pathological findings. Radiol Med. 2017;122:902-8. 
9. Zhao W, Zhong Z, Xie X, Yu Q, Liu J. Relation between chest CT findings and clinical conditions of coronavirus disease (COVID19) pneumonia: a multicenter study. AJR Am J Roentgenol. 2020;214:1072-7.

10. Shi H, Han X, Jiang N, Cao Y, Alwalid O, Gu J, et al. Radiological findings from 81 patients with COVID-19 pneumonia in Wuhan, China: a descriptive study. Lancet Infect Dis. 2020;20:425-34.

11. Hani C, Trieu NH, Saab I, Dangeard S, Bennani S, Chassagnon G, et al. COVID-19 pneumonia: A review of typical CT findings and differential diagnosis. Diagn Interv Imaging. 2020;101:263-8.

12. Plenzig S, Bojkova D, Held H, Berger A, Holz F, Cinatl J, et al. Infectivity of deceased COVID-19 patients. Int J Legal Med. 2021. https://doi.org/10.1007/s00414-021-02546-7.

13. American College of Radiology. ACR recommendations for the use of chest radiography and computed tomography (CT) for suspected covid-19 infection, 11 March 2020. https://www.acr.org/Advocacyand-Economics/ACR-Position-Statements/Recommendations-forChest-Radiography-and-CT-for-Suspected-COVID19-Infection. Accessed 1 Apr 2021.

14. RANZCR. Guidelines for CT chest and chest radiograph reporting in patients with suspected COVID-19 infection. 24 April 2020. https://www.ranzcr.com/our-work/coronavirus/position-statementsand-guidance. Accessed 1 Apr 2021.

15. Royal College of Radiologists. RCR position on the role of CT in patients suspected with COVID-19 infection. 12 March 2020. https://www.rcr.ac.uk/college/coronavirus-covid-19-what-rcrdoing/clinical-information/role-ct-chest/role-ct-patients. Accessed 1 Apr 2021.

16. Canadian Association of Radiologists. Canadian Society of Thoracic Radiology and Canadian Association of Radiologists' Statement on COVID-19. 26 March 2020. https://car.ca/news/canadian-societyof-thoracic-radiology-and-canadian-association-of-radiologistsstatement-on-covid-19. Accessed 24 Apr 2021.

17. Xie X, Zhong Z, Zhao W, Zheng C, Wang F, Liu J. Chest CT for typical coronavirus disease 2019 (COVID-19) pneumonia: relationship to negative RT-PCR testing. Radiology. 2020;296:E41-5.
18. Schweitzer W, Ruder T, Baumeister R, Bolliger S, Thali M, Meixner E, et al. Implications for forensic death investigations from first Swiss post-mortem CT in a case of non-hospital treatment with COVID-19. Forensic Imaging. 2020;21:200378.

19. Ducloyer M, Gaborit B, Toquet C, Castain L, Bal A, Arrigoni $\mathrm{PP}$, et al. Complete post-mortem data in a fatal case of COVID19: clinical, radiological and pathological correlations Int J Legal Med. 2020;134:2209-14.

20. Cittadini F, De-Giorgio F, Cina A, Pascali VL. Reliable postmortem computed tomography scan diagnosis of COVID-19 Pneumonia. Am J Forensic Med Pathol. 2020;41:239-40.

21. Barisione E, Grillo F, Ball L, Bianchi R, Grosso M, Morbini P, et al. Fibrotic progression and radiologic correlation in matched lung samples from COVID-19 post-mortems. Virchows Arch. 2021;478:471-85.

22. Williams AS, Dmetrichuk JM, Kim P, Pollanen MS. Postmortem radiologic and pathologic findings in COVID-19: The Toronto experience with pre-hospitalization deaths in the community. Forensic Sci Int. 2021;322:110755.

23. Bai HX, Hsieh B, Xiong Z, Halsey K, et al. Performance of radiologists in differentiating COVID-19 from Non-COVID-19 viral pneumonia at chest CT. Radiology. 2020;296:E46-54.

24. Wichmann D, Sperhake JP, Lütgehetmann M, Steurer S, Edler C, Heinemann A, et al. Autopsy findings and venous thromboembolism in patients with COVID-19: A prospective cohort study. Ann Intern Med. 2020;173:268-77.

25. Sperhake JP. Autopsies of COVID-19 deceased? Absolutely! Leg Med (Tokyo). 2020;47:101769.

Publisher's Note Springer Nature remains neutral with regard to jurisdictional claims in published maps and institutional affiliations. 